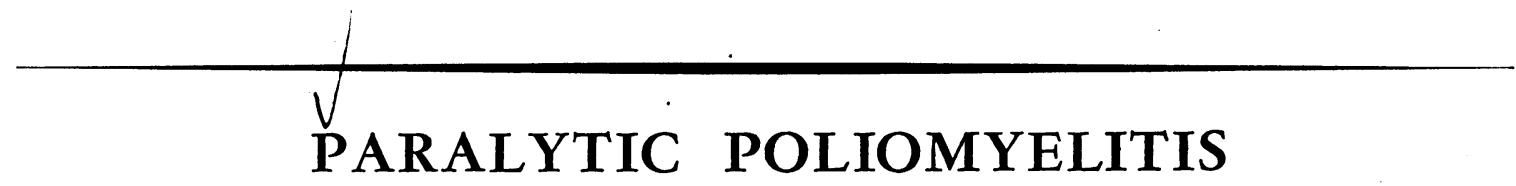

By A. Graham Apley, F.R.C.S.

Consultant Orthopaedic Surgeon, Rowley Bristow Orthopaedic Hospital; Assistant to the Department of Orthopaedics, St. Thomas's Hospital, London

\section{Introduction-The Stages of Poliomyelitis}

The terms used to describe the successive stages of poliomyelitis vary widely. For the purpose of this article the disease will be considered to pass through five stages (Ritchie Russell, r949), which may be briefly summarized as follows:

\section{Prodromal (Inv:asion) Stage}

Invasion probably occurs via the pharynx. A ' minor illness' results with fever, malaise, sore throat and gastro-intestinal upset. This stage lasts only 24 to 48 hours and is usually so trivial as to be recognised only in retrospect. The only feasible treatment is prophylaxis by preventive inoculation and precautionary isolation.

\section{Pre-paralytic (Meningitic) Stage}

The virus has by now penetrated the central nervous system, and is there multiplying. Clinically there may be a ' major illness,' usually abrupt in onset, with fever, headache, vomiting, stiffness of neck and back, and pain in the trunk and limbs. The patient is in a critical phase, hovering for some days between complete recovery and what may be disastrous paralysis. He must be rested immediately, and the rest enforced and complete, because fatigue may precipitate paralysis and undoubtedly increases its severity. (Ritchie Russell, 1947, Horstmann, 1950).

\section{Paralytic (Acute) Stage}

The virus attacks and destroys anterior horn cells. Soon after the major illness paralysis appears and is quickly maximal in extent. There is, in addition, spasm and tenderness of muscles. The essentials of treatment are rest, assisted respiration if necessary, and the relief of discomfort.

\section{Convalescent (Recovery) Stage}

The virus is dead, but has destroyed a variable number of nerve cells. The patient is no longer ill in himself, and paralysis to some extent recovers. The patient's recovery of function is assisted by physical treatment and by training, while intermittent splintage is often required.
5. Definitive (Chronic) Stage

There is permanent residual paralysis with deformity, flailness and trophic changes.

As a rule, only the last three of these five stages concern the surgeon, and these will now be described.

\section{The Paralytic or Acute Stage}

Pathology. Predominantly, the virus of poliomyelitis attacks the anterior horn cells. It is likely that 'fatigued' cells fall the most ready victims. The attacked cells undergo chromatolysis, and the disintegrating nuclear fragments are rapidly phagocytosed, so that the cell virtually disappears in a few days. Many cells, however, undergo chromatolysis but escape phagocytosis (Bodian, 1948) and these cells regain a normal microscopic appearance within a remarkably short space of time, usually four to five weeks (Edds, 1950). Cells which recover were presumably less fatigued, less virulently attacked, or merely damaged by oedema.

Virus and oedema may damage not only cells in the anterior horn, but also antero-lateral horn cells, and the so-called inter-nuncial cells concerned with short linkages within the cord.

Clinical Features. The paralytic stage is arbitrarily defined as lasting from the onset of definite muscle paralysis (usually one to five days after the ' major illness') until all pain and spasm have disappeared. It is not to be supposed that paralysis is common, even after the 'major illness,' and the modern view (W.H.O. Report, 1954) is that paralysis, whether bulbar or spinal, is an infrequent complication of a common disease. Occasionally, on the other hand, the pre-paralytic stages have passed unnoticed, and paralysis is one of the first presenting features.

Paralysis varies enormously in extent and distribution, but is always flaccid in type. Spinal involvement may paralyse limb muscles, the paralysis being characteristically asymmetrical in distribution, with the lower limbs more often involved than the upper; more immediately important is paralysis of the diaphragm, intercostal 
muscles and accessory muscles of respiration, leading to dyspnoea and, rarely, to death.

In the spinal type of paralytic poliomyelitis the patient's mind remains unclouded, even though he is irritable and apprehensive; the sudden development of flaccid paralysis in an alert patient is strong presumptive evidence of poliomyelitis. Some of the spinal muscles are painful, tender, and resentful of rough handling and of stretching. These muscles are often said to be in 'spasm,' a muchabused word in the vocabulary of poliomyelitis. Almost certainly the spasm is simply a reflex guarding, to prevent nerve roots from being stretched (Mitchell, 1954). Spasm and pain are distressing symptoms, and often associated with sweating and hyperaesthesia. Sometimes there is a temporary retention of urine.

The bulbar type of disease, which appears to be increasingly frequent, may attack various cranial nerves, of which the most vital are those supplying the laryngeal and pharyngeal muscles. If these are damaged, a rattling noise develops in the throat of the distressed patient who is unable to cough away excess mucus, and speaks in a feeble whisper; dysphagia and dyspnoea are.also common. Bulbar palsy is a sinister condition; it demands urgent diagnosis and urgent treatment.

\section{Treatment}

The patient must be kept alone (isolated), alive (by artificial respiration if necessary), and aerated (with a clear airway); he should be relieved (of pain) rested (by intermittent splintage) and relaxed (by reducing 'spasm'). These points require separate consideration.

Poliomyelitis is an infectious disease, not only during the pre-paralytic, but also during the early weeks of the paralytic stage. Droplet infection should be prevented, and the faeces, which also contain the virus, disposed of as in enteric fever; in fact the patient is barrier-nursed. Quarantine of contacts is a rarely attainable ideal, but children who have been intimate contacts should certainly be kept at home, and they should avoid overexertion, though how this latter can be achieved in an active child I have never understood.

If respiratory muscles are weak the patient must be helped to breathe. Until recently this object was achieved by means of an 'iron lung.' The patient's body, but not his head, was enclosed in an air-tight coffin-like box, and the air pressure within the box alternately raised and lowered by means of a pump. The apparatus is cumbersome and makes nursing difficult; moreover, if there is also bulbar paralysis, the forced inspiration is liable to suck mucus into the bronchi. A simpler way to assist breathing is to perform tracheotomy, connecting the tracheal tube to a breathing appara- tus (like a Boyle's anaesthetic machine), and to control this apparatus by a pump.

To maintain a clear airway is of special importance in bulbar palsy. The patient's position should be frequently (but gently) changed, so that gravity assists the drainage of mucus; intermittent suction is also useful (Lassen, 1950). If there is bilateral paralysis of the laryngeal abductors a tracheotomy is essential.

The patient is apprehensive, alert, and in pain, so that analgesics and sedatives must not be withheld. He should be rested on a flat bed with fracture boards covered by a rubber mattress. One pillow is usually sufficient, and no elaborate splints should be used. If the arms are paralysed they may be rested on pillows or suspended in Guthrie Smith slings. The wrists can be supported in a cock-up splint, and the thumb held opposed by an elastic garter. The patient lies flat with the hips extended, and a small pad or pillow prevents the knees from hyper-extending. A board for the feet to rest on is usually sufficient to prevent foot drop. The emphasis is on rest in a natural position, and on the avoidance of cumbersome splints. To discourage the development of contractures, not only is the posture changed at frequent intervals (nurses as well as physiotherapists should be trained to do this) but affected limbs are twice a day moved passively through their full painless range. Such movements should not be forced and must never hurt the patient.

It has for long been realised that passive movement of affected limbs is often painful, evoking ' spasm ' and cramps. How may these be avoided? Some American workers (Ranschoff, 1948) have employed curare-like relaxants, but they do not abolish pain and are not advised. Probably the best method is that of Sister Kenny and her disciples; the affected limbs are wrapped in hot moist packs, which are comforting and usually permit increased passive movement (Bingham, 1943). Sister Kenny's methods and, indeed, her very name, engender fierce controversy, probably because although she was unqualified, she treated patients better than did most doctors. Her methods were based upon quite unsound pathology, but they worked. Their success was largely due to the abandonment of elaborate splintage, the relief of pain and spasm, and the regular employment of gentle passive painless movements; all these in addition to her boundless enthusiasm.

\section{The Convalescent or Recovery Stage}

Pathology. The dead cells, in which chromatolysis was succeeded by phagocytosis, are now converted into scar tissue; their axons undergo Wallerian degeneration and the muscle fibres which these axons supply are paralysed completely and 
without possibility of recovery. Cells which were damaged but not destroyed recover fully and speedily, so that the muscles they supplied are capable of functioning again within a few weeks. The contrast between irrevocable destruction and rapid return to potential normality is marked; and yet the clinical process of improvement takes place gradually over a period of many months. Such slow improvement is due to hypertrophy of surviving nerve cells and muscle fibres, a process which is assisted and hastened by training. For a limb to be paralysed severely and permanently, about two-thirds of the 12,000 anterior horn cells supplying it must have been killed (Bodian, r948).

Clinical Features. The disease is said to be in the convalescent stage when the muscles are no longer painful or tender, which occurs at about six to twelve weeks from the onset of paralysis, though occasionally it lasts much longer. Quite arbitrarily the stage is held to last two years.

The patient is not ill. Headache, malaise and pain are absent; and so is muscle spasm, in spite of Sister Kenny's assertions to the contrary. Permanent paralysis of any muscle group leads, of course, to weakness; but this weakness is usually manifested in one of two ways. First, if the muscles controlling a joint are all paralysed, a condition conveniently called balanced paralysis, the joint becomes flail. Second, unbalanced paralysis, in which one muscle group is more severely involved than its opponent, results in deformity at the joint; stronger muscles overcome weaker and with the passage of time may lose their extensibility, so that the deformity becomes fixed. Some authorities believe that deformity may also result from contracture in skin and in subcutaneous fibro-elastic tissues, or from the persistence of untreated spasm in muscles; proof for these views is lacking, but it is not possible to explain all deformities simply on the grounds of unopposed or unbalanced muscle action.

In addition to weakness, wasting, and deformity or flailness, trophic changes appear, the limb becoming cold and blue. These trophic changes are usually thought to result from venous pooling, a sequel to the paralysis, and probably this is the true explanation; but sometimes the trophic changes are disproportionately greater than the paralysis, possibly because of paralysis of the actual blood vessel walls due to damaged antero-lateral horn cells.

\section{Treatment}

Treatment in the convalescent stage is most simply considered under four headings:

I. The patient is fit and should be got up. He should be got up and about as soon as possible, if necessary with splints, if necessary with crutches, if necessary in a wheel chair, but up at all costs. Only two factors should be allowed to stand in the way; first, if respiration still needs assistance, when the process of weaning the patient from assisted breathing may have to be slow; and second, if there is unbalanced trunk paralysis, for this carries a high risk of scoliosis which may quickly become severe. If scoliosis threatens, the patient should be kept recumbent until hypertrophy has occurred, or until a back support controls the potential deformity.

2. Some muscle groups remain paralysed, and splintage is often required. Paralysis does not by any means always demand splintage. Unbalanced paralysis, however, may need splintage to prevent the surviving muscles from shortening and producing fixed deformity; and balanced paralysis may require splintage to provide stability. Any splints employed should be removed at least twice a day while the affected joints are moved passively through their full range.

3. Some muscle groups are 'recovering' and require exercise. Gradual ' recovery' is due to hypertrophy, a process which should be encouraged with all speed and energy. It is pointless to exercise muscles which have already recovered fully, and it is futile to attempt to train muscles which are paralysed completely. Treatment must concentrate upon muscles which are weak enoughco to need training, yet active enough to benefit from it. Training should begin early, and should quickly become strenuous (Mead, 1950). It is only during the earlier, still active stages of the disease that exertion is dangerous and rest essential. Once the convalescent stage is reached the attitude of doctor, physiotherapist and patient should undergo a complete volte-face. Rest is now eschewed; the patient is coaxed, cajoled, persuaded and, if necessary, bullied into increasing activity and power, as if he were a boxer training for a fight. It is during this training programme that charts of muscle power are helpful, the strength of each group being recorded thus:

$$
\begin{aligned}
& \text {.... no activity. } \\
& \text { I... . . flicker. } \\
& 3 \ldots \text { contraction, not powerful enough to act } \\
& \text { against gravity. } \\
& \text {.... sufficient power to act against gravity. } \\
& \text { resistance. }
\end{aligned}
$$

Once it is apparent that a particular group will never achieve useful power (three or better), the physiotherapist may permit and even encourage the development of trick movements, teaching muscles to assume unfamiliar functions.

4. The limb is trophic and requires 'coddling.' It is difficult or impossible to prevent trophic 
changes from developing, but their effects must be minimized if training is to achieve its effect. The essential is never to allow the limb to become cold. Keep the bed warm, the room warm and wellventilated, and the limb itself warm; woollen stockings, or two pairs, are helpful. Exercises in a warm swimming pool, sometimes dignified by the name of hydrotherapy, are also useful: the water makes the patient feel light in body and light in spirit.

\section{The Definitive or Chronic Stage}

Pathology. The dead nerve cells have been replaced by scar tissue. Paralysed muscles are wasted, and if a limb is severely involved the bones become slender and decalcified. Moreover, the limb does not grow normally, so that paralysis during childhood leads to relative shortening.

Clinical Features. The patient is completely fit except for his paralysis. Paralysis is lowermotor-neurone in type, the muscles being flaccid and tendon reflexes diminished. The affected limb (or limbs) may look blue, is wasted and often short and deformed. There are frequently extensive chilblains and the skin is cold to the touch. When a badly paralysed limb is picked up, it has a peculiar 'floppy' feel which, in the presence of normal skin sensation, is almost characteristic of poliomyelitis.

As far as the actual paralysis is concerned, the patient may be affected in three ways: first, as isolated weakness, in which there is inability to perform an individual action; second, there may be unbalanced paralysis of the muscles controlling a joint, in which case there is not only weakness, but possibly also fixed deformity; and, third, there may be complete or balanced paralysis resulting in a flail joint. Most joints have two main movements, one assisted by, and one opposed to, gravity. Paralysis of the anti-gravity muscles is the more disabling and more liable to lead to fixed deformity.

\section{Treatment-Principles}

Further true recovery is, by definition, impossible. Attention must now be concentrated on minimizing the functional effects of the paralysis, largely by apparatus or by surgery. The main principles are:

I. First, to consider the patient as a whole. It is pointless to embark upon an extensive programme of reconstructive surgery in a limb if the patient's paralysis is so severe and widespread that he will be unable to take advantage of any localized improvement.

2. Next, to consider the limb as a whole. No splint or operation should be employed to improve the function of a portion of a limb unless the use- fulness of the limb as a whole is also thereby increased. For example, reconstructive surgery to the hand may be a pointless luxury if the patient is unable to put his hand to its task. Again, it may be foolish to operate upon a foot, apparently improving its function, if that function cannot be utilized because the patient has still to wear a caliper in order to control his knee. While considering the limb as a whole, the problems of trophic changes and shortening may require attention. These problems are more frequent and important in the lower than in the upper limb.

3. Finally, to consider the individual parts. Balanced paralysis results in a flail joint. In the lower limb stability is essential, and the flailness must be dealt with by splintage or by arthrodesis. Unbalanced paralysis leads to deformity, and if this proves disabling it may be practicable to divide the strong deforming muscle through its tendon and to re-attach the tendon more usefully; not only is deformity overcome, but balance may be restored. The re-routing of muscles may also be used in treating weakness of individual actions, particularly in the upper limb where such actions are often more important than stability. Transplanted tendons should, if possible, be anchored to bone. It is easier to learn to use the transplanted tendon and muscle if it is a synergist of the paralysed muscle.

\section{Survey of Treatment in the Definitive Stage Trunk}

Unbalanced paralysis, in which one side of the back or belly muscles is stronger than the other, leads to scoliosis. The scoliosis is liable to become severe, because gravity assists the stronger group, and the weaker group becomes stretched and still weaker. Spinal jackets and supports are of some help in minimizing deformity, but severe imbalance is rarely controlled satisfactorily, and it is sometimes wise to fuse a portion of the spine after correcting the curve.

Balanced paralysis leads to a floppy trunk, but not to a fixed deformity. External supports can provide reasonable stability; they are of greater benefit than with unbalanced paralysis, for in the latter it is difficult to oppose a constant unilateral force.

\section{Shoulder}

Unbalanced paralysis. Weak adductors matter little, for once the limb has been abducted, gravity can bring it down again; therefore no deformity and little disability result. Weak abductors, however, render the limb almoșt useless. Moreover, the strong unopposed adductors, aided by gravity, lead to fixed adduction deformity which may 
require operative correction. Loss of ability to abduct may be overcome as described below.

Balanced paralysis. Abductor action can be restored to the limb by arthrodesing the shoulder -providing that the scapular muscles are powerful. If the trapezius, rhomboids and serratus magnus are strong, they can act on the 'scapulohumerus' enabling the limb to be placed and stably maintained in useful positions. The joint should be arthrodesed in $70^{\circ}$ of abduction and $30^{\circ}$ forward of the coronal plane. The operation is a useful one for abductor paralysis, whether balanced or unbalanced; it should, however, only be performed if the hand is sufficiently useful to take advantage of the improvement.

\section{Elbow}

Unbalanced paralysis matters as a rule only if the forearm flexors are paralysed, for gravity can replace the extensors. The exception is in the patient who has to use crutches, for elbow extensors are then necessary. Normally, however, if only the extensors are powerful, it is reasonable to re-attach them so that they will act as flexors; even though the resulting power is unlikely to permit weight to be lifted, it may be sufficient to lift the hand to useful positions.

Balanced paralysis leads to a flail elbow. Again it is flexion that matters most. An intact pectoralis major can be dis-inserted and attached to the biceps. Failing this, it is sometimes possible to advance the origin of the wrist flexors higher up the humerus. Even if there are no muscles to stabilize the joint it is better for the patient to wear a moulded splint, which holds the elbow bent at a useful angle, rather than to have the joint arthrodesed. Modern polythene splints are light, clean. and comfortable.

\section{Wrist and Hand}

Unbalanced paralysis of wrist flexors is not much of a handicap, for gravity opposes the extensors. But paralysed extensors in the presence of strong flexors leads to fixation of the wrist in the flexed position, a poor position for function. The deformity can, to some extent, be prevented by splintage but once deformity has occurred the wrist flexors should be re-routed to act as extensors.

Balanced paralysis of flexors and extensors gives a flail wrist; but the wrist is not totally flail unless the long finger muscles are also paralysed. A flail wrist may be stabilized by a cock-up splint or by an arthrodesis. Operation, however, is not often indicated, for it should only be performed if the fingers function well enough to take advantage of the stability, and if the fingers do function well the wrist usually has sufficient stability already.

Paralysis of the thumb and fingers are sometimes benefited by surgery. The hand as a whole works as a vice and as pincers; each of these functions should be considered. For the vice action finger flexors are required, preferably aided by wrist extensors. It is sometimes advisable to arthrodese the wrist and utilize any acting wrist muscles to re-inforce finger flexion. It should be noted that the arthrodesis serves two purposes; it stabilizes the wrist, and it also ' liberates ' muscles which can be given more important tasks. The pincer action of the hand demands a strong opponens pollicis; if this muscle is paralysed a substitute can be provided by detaching the flexor sublimis from one finger, winding the tendon round that of flexor carpi ulnaris (which acts as a pulley) and inserting the sublimis into the radial side of the thumb. Excellent opposition is obtained in this way.

\section{Hip}

Unbalanced paralysis is chiefly of importance when it affects the glutei and the tensor fascia lata. If, during the paralytic stage, the patient has been treated sitting up instead of lying flat, fixed flexion and adduction are liable to develop at the hip. Once present this deformity is difficult to overcome, and its correction may require stretching and plaster, or even surgical division of the flexors. Occasionally, unbalanced paralysis of the adductors and extensores leads to dislocation of the hip, which makes the inevitable Trendelenburg limp even worse. Hip dislocation in poliomyelitis is very difficult to deal with and arthrodesis is occasionally worth consideration.

Balanced paralysis of the hip muscles leads to flailness. Flexion can be achieved by thrusting the trunk forwards in walking, so that gravity helps the lower limb forwards into a flexed position; but extension and abduction cannot be mimicked, and when weight is taken on the limb a severe Trendelenburg dip occurs. No satisfactory treatment is known. A method sometimes used is to fit a caliper attached by a hinged bar to a pelvic band; and to incorporate an adduction lock in the bar. The apparatus is cumbersome and not very efficient. Arthrodesis of the hip does at least provide stability, but is technically difficult to achieve, and a stiff hip is a great nuisance if the knee muscles are also paralysed.

\section{Knee}

Unbalanced paralysis of the knee flexors is not often disabling, for the straight leg can be lifted by flexing the hip while the quadriceps contracts, and gravity flexes the knee as soon as the quadriceps is allowed to relax. Only rarely does a genu recurvatum develop and necessitate a caliper with a Jones's knee brace.

Unbalanced extensor paralysis is more disabling 
and may result in fixed flexion deformity at the knee. It is a little unfashionable to transpose the hamstrings to the quadriceps, but the operation is well worth while; not only are the deforming muscles divided, but sometimes the reinforced quadriceps muscle is strong enough to stabilize the knee without a caliper.

Balanced paralysis of the knee flexors and extensors is common and results in flailness. The simplest treatment is to provide a caliper, which should be hinged at the knee to prevent the straight leg from being a nuisance in buses; the hinge must have a simple locking device to prevent the caliper from buckling when the patient stands. Women sometimes prefer to dispense with the caliper and have the knee arthrodesed; the individual must weigh the inconvenience of a permanently stiff leg against the elegance of a well-stockinged one. Arthrodesis is probably best performed with the knee at $180^{\circ}$, but there is a modern trend towards arthrodesis in $20^{\circ}$ or so of flexion, providing that the disease has not shortened the limb. The flexion makes the leg less of an obstruction to other people, and allows it to swing through in walking.

It is by no means always necessary to treat quadriceps paralysis with either splint or operation; for if the ankle is in fixed equinus, when the patient takes weight the equinus foot forces the knee into slight hyperextension, and in this position the knee is often stable enough to permit safe walking.

\section{Ankle}

Foot paralyses tend to be combined in a wide variety of ways; moreover the long muscles of the toes act also on the ankle and sub-taloid joints. Despite these complicating factors it is simplest to consider the foot as three separate joints: ankle, sub-taloid, and metatarso-phalangeal (the term sub-taloid is used to mean the sub-taloid and midtarsal complex).

Unbalanced paralysis at the ankle joint is chiefly of importance when the dorsiflexors are weak. A toe-raising spring or back-stop attached to a leg iron can prevent foot-drop; but if the plantar flexors are strong enough and unopposed, they pull the foot into fixed equinus which requires correction by stretching and plaster, or by division of the deforming tendon. It should be a rule that when any tendon is divided to overcome fixed deformity in poliomyelitis that tendon should be inserted usefully elsewhere.

Strong ankle dorsiflexors with weak plantarflexors is less often disabling or deforming, because gravity assists plantarflexion. If, however, imbalance is gross, fixed calcaneus deformity (usually associated with cavus) develops, and requires surgical correction. Elmslie's operation (1934) is satisfactory for this purpose. It consists of a twostage sub-taloid fusion, the second stage being by a posterior approach and combined with tendon transplantation.

Balanced paralysis of ankle muscles, especially if combined with weak toe muscles, leads to flailness. Stability is readily provided by a below-knee caliper with a toe-raising spring attached to the shoe. It is often possible, however, to dispense with apparatus by performing Lambrinudi's operation (Lambrinudi, 1927); the sub-taloid and midtarsal joints are arthrodesed and a segment cut off the talus so that with the talus in gross equinus the foot fits on to it in only slight equinus. In its fully equinus position the talus locks into the ankle mortice and cannot plantarflex further. The slight equinus of the foot helps to compensate for any associated quadriceps paralysis (see above). The idea behind Lambrinudi's procedure is a brilliant one, and the operation finds wide application in foot paralysis, permitting deformity to be overcome and stability to be achieved. It is important to note that arthrodesis of the ankle joint is not required in poliomyelitis.

\section{Sub-taloid Foint}

Unbalanced paralysis may affect the invertor muscles so that when the foot is dorsiflexed it pulls up into valgus (eversion), and in time develops a fixed valgus deformity. It is important to balance the foot, and to balance it as early in the disease as possible-even during the convalescent stage. The strong evertors should be re-inserted into such a position that they pull the foot up straight. But if weak invertors are combined with weak ankle dorsiflexors, as is commonly the case, a Lambrinudi's operation is often the best procedure.

Similarly, unbalanced evertor paralysis results in the foot being pulled up into varus (inversion) and may lead to fixed varus deformity. Balance may be achieved by a toe-raising spring attached to the outer side of the shoe, or by a tendon transplant. Frequently the associated paralyses are such that Lambrinudi's operation is required. It should be noted, however, that any sub-taloid fusion is likely to fail if a strong but unbalanced muscle is left intact. Such a muscle (or its tendon) should be divided and, as usual, employed usefully elsewhere.

Balanced paralysis of the sub-taloid joint leads, not to a fixed deformity, but to a floppy unstable foot. To some extent the flailness can be controlled by a below-knee caliper, but arthrodesis of the sub-taloid mid-tarsal joint is the most satisfactory procedure. Dunn's triple arthrodesis provides excellent sub-taloid stability, but often Lambrinudi's operation is more suitable because of associated foot drop. 
Toe Foints

Unbalanced paralysis of the muscles controlling the toes is of importance only when the long muscles overpower the short. The result is a bunching-up of the whole foot into claw foot and claw toes-deformities which soon become fixed. The metatarsalgia which inevitably results may be helped by suitable padding, supports and footwear, designed to distribute pressure evenly. Lambrinudi advised arthrodesing all the inter-phalangeal joints in the straight position, and re-inserting the long extensor tendons into the metatarsal necks, an operation which is laborious but has good results.

Balanced paralysis of toe muscles is of little importance except for its frequent association with other foot weaknesses. It is of interest that weak leg and foot muscles rapidly result in gross flat foot, but only if the external rotators of the hip are also paralysed; for these rotators are partially inserted into the tibia (via the ilio-tibial tract) and, when they act while the foot is anchored by body weight to the ground, they twist the tibia outwards and so lift the inner border of the foot.

\section{Trophic Changes and Shortening.}

In the upper limb trophic changes usually occur only with gross paralysis, and shortening is of no account. In the lower limb, however, a cold, blue limb with extensive chilblains, is distressingly frequent, and shortening may be both severe and disabling. The trophic changes are impossible to prevent and difficult to treat. Two pairs of socks or stockings and fur-lined boots are helpful, but once chilblains develop they are liable to ulcerate and only to heal temporarily in the summer. It is important, not so much to warm cold feet, as never to allow them to become cold-a counsel of perfection in England. Lumbar sympathectomy has intermittently evoked staunch advocacy but there is a widespread feeling that the operation is helpful only for two or three years, after which the trophic changes recur. The combination of skin-grafting with sympathectomy certainly allows an otherwise intractable trophic ulcer to be satisfactorily healed, and the operation could perhaps be employed more often than it is at present.

Shortening can be compensated for by a raised shoe, and this is often the best method. Actual lengthening may be achieved by oblique division of the femur, or of the tibia and fibula, and treating the osteotomy with traction so that union occurs in an elongated position. The procedure is dangerous, since it is liable to many complications, and is rarely performed. An alternative procedure is to shorten the longer leg, which may be achieved before growth is completed by fixing metal staples or blocks of bone across the growth discs. The operation is simple and free from complications, but there is a reasonable reluctance to interfere with a patient's remaining sound leg. It may be that in future a simple and safe method of lengthening a bone will be achieved, for it has been shown (Trueta, 1953) that if the portion of the bone medulla which includes the nutrient artery is blocked (by disease, injury or experiment), the juxta-epiphyseal arteries hypertrophy and the bone subsequently grows longer.

\section{BIBLIOGRAPHY}

BINGHAM, R., F. Bone Ft. Surg., 25, (3), 647.

BODIAN, D. (1948), 'Pathological Anatomy in Poliomyelitis,' Lipincott, Philadelphia.

EDDS, M. V. (1950), Ұ. Comp. Anat., 93, 259.

ELMSLIE, R. C. (I934), 'In Modern Operative Surgery', Cassell $\&$ Co. Ltd., L.ondon, (quoted by Cholmeley, J. A., $\mathcal{F}$. Bone $\boldsymbol{f} t$. Surg., 35b, (I), 46).

LAMBRINUDI, C. (1927), Brit. f. Surg., 15, 193.

LASSEN, H. C. A., (1953), Lancet, 37.

MEAD, S. (1950), F. Amer. Med. Ass., 144, 458.

MITCHELL, G. P. (1954), Lancet, 18.

RANSOHOFF, N. S. (1950), Med. Clinics of N. America, 34, 562 RITCHIE RUSSELL, W. (1947), Brit. Med. F., 1019.

RITCHIE RUSSELL, W. (1949), Brit. Med. F., 465.

RITCHIE RUSSELL, W. (1952), 'Poliomyelitis,' Edward Arnold \& Co., London.

TRUETA, J., Bull. Hosp. Ұt. Dis., New York, 14, 2, 147.

WORLD HEALTH ORGANISATION (1954), Technical Report on Polionyelitis, $8 \mathrm{r}$.

\section{RUTHIN CASTLE, NORTH WALES}

A Clinic for the diagnosis and treatment of Internal Diseases (except Mental or Infectious Diseases)." The Clinic is provided with a staff of doctors, technicians and nurses.

The surroundings are beautiful. The climate is mild. There is central heating throughout. The annual rainfall is $\mathbf{3 0 . 5}$ inches, that is, less than the average for England.

The Fees are inclusive and vary according to the room occupied.

For particulars apply to THE SECRETARY, Ruthin Castle, North Wales.

Telegrams: Castle, Ruthin.

Telephone: Ruthin 66 